PSICOLOGIA Vol. VIII № 21990

\title{
ANOREXIA NERVOSA UN ESTUDIO DE CASOS
}

Lillyana Zusman*

La Anorexia Nervosa es un trastorno de alimentación que se define (etimológicamente) como una "pérdida nerviosa del apetito". Se caracteriza por la actitud consciente, voluntaria y rotunda de los sujetos de no comer. Existe en ellos/as la firme convicción de tener un exceso de peso que intentan modificar por vía de la inanición.

A partir del estudio de casos, se propone la distinción entre una Anorexia Nervosa Estructural -aquélla en la que predomina el conflicto intrapsíquico primario y arcaico, y que manifiesta una conducta aislada y retraída- y una Anorexia Nervosa Reactiva, aquélla en la que predomina un conflicto intrafamiliar algo más posterior $\mathrm{y}$, por lo tanto, una personalidad más abierta y compatible con el medio.
Anorexia Nervosa is an Eating Disorder defined (etimoligacally) as a "nervouse loss of apetite". It is characterized by a conscious, voluntary and categoric attitude of the sick patient to refrain from eating. He/she has a firm conviction of having excess weight which they try to modify by starvation.

Through a case study, a distinction between two types of Anorexia Nervosa is proposed: a Structural Anorexia Nervosa -in which an intrapsyhic, primary, arcaic conflic prevails, leading to an isolated, withdrawal conduct- and a Reactive Anorexia Nervosa, in which a later intrafamilial conflict prevails leading, therefore, to a more open personality, compatible with the enviroment.

* Pontificia Universidad Católica del Perú. 

La Anorexia Nervosa es uno de los trastornos de la alimentación que se define etimológicamente como una "pérdida nerviosa del apetito". ${ }^{1}$ Se caracteriza por la actitud consciente, voluntaria y rotunda de los sujetos enfermos de no comer, ya que existe en ellos la firme convicción de que presentan sobrepeso y de que es vía la inanición como modificarán su cuerpo grueso y pesado. Los síntomas de esta enfermedad no tienen un correlato físico. El eje del diagnóstico se articula en tomo a la actitud férrea de los pacientes de no comer, por una parte, y a la convicción de que poseen un cuerpo adecuado cuando, en realidad, su cuerpo es sumamente delgado. Se trata de una delusión corporal (Slade, 1984).

La Anorexia Nervosa es una enfermedad antigua. Existen datos que confirman su existencia desde la Edad Media. Sin embargo, las primeras descripciones de carácter clínico se hicieron en Inglaterra durante los siglos XVII y XVIII al calificar a la enfermedad como una "atrofia nerviosa" (Slade, 1984). A lo largo del siglo XIX, se vínculo a la Anorexia Nervosa con la Histeria y se reconoció la participación de la familia en el origen de la misma. Asimismo, se la diferenció de la Anorexia Gástrica, la cual se asoció, más bien, a problemas gástricos y a la mala absorción de alimentos. A principios del siglo XX, se presentaron versiones diferentes acerca de la etiología y de las características de la enfermedad (Janet, 1903; Thomas, 1909; Simmonds, 1914). A partir de mediados del siglo XX se formularon hipótesis psicoanalíticas diversas que contribuyeron a aclarar la naturaleza intrapsiquica de la enfermedad e hipótesis psiquiátricas que posibilitaron la descripción de un diagnóstico diferencial para establecer los límites claros entre la Anorexia Nervosa, la Esquizofrenia y la Depresión. En la década del 70, finalmente, el aporte sistémico (Minuchin, 1978, Selvini Palazzoli, 1974) ofrecio una nueva perspectiva de comprensión de la enfermedad al plantearla como un problema interpersonal que se origina $v$ que repercute en el sistema familiar.

1. Dentro del rubro de los trastornos de la alimentación, el DSM III considera à la Anorexia Nervosa y a la Bulimia. 
A partir de investigaciones realizadas en diferentes niveles de conceptualización, se ha concluido que la Anorexia Nervosa es una enfermedad que no presenta una causa específica y discreta sino que, más bien, se trata de una coyuntura en la que interactúan un conjunto de cadenas complejas de eventos que precipitan la enfermedad. Hsu (1983) considera que, por el momento, no es del todo importante cuestionarse acerca de las causas de la enfermedad porque se acepta la pluricausalidad de la misma, sino qué, más bien, es importante averiguar la coyuntura adecuada sobre la cual se presenta la misma para que a partir de este punto se puedan plantear estrategias de intervención adecuadas que sean efectivas tanto sobre el paciente identificado como sobre su familia.

La corriente psicoanalítica (Brunch, 1974, 1981, 1983; Crisp, 1970, 1974 a y b: Garfinkel \& Gardner, 1982; Mushatt, 1982,1983; Wilson, 1982) en tanto favorece la compresión intrapsíquica de la enfermedad y la corriente sistématica (Minuchin, 1978; Selvini Palazzoli, 1974) en tanto enfoca a la misma dentro de un contexto interpersonal-familiar posibilitan, en conjunto, una comprensión global de la enfermedad. Sin embargo, es importante tener en consideración que existe un conjunto de factores socio-culturales y psicobiologicos que operan también como determinantes de la enfermedad.

La teoría psicoanalítica considera que el nacimiento biologico del infante no coincide con su nacimiento psicológico (Mahler, 1975). Mahler vincula el nacimiento psicológico a la consecusión de los procesos de separación-individuación y a la consecuente capacidad del niño para establecer una relación con un mundo de realidad tanto en lo que se refiere a las relaciones de objeto como a la relación con su propio cuerpo.

Durante la primera etapa de la vida, mientras el niño satisface sus necesidades corporales y va tomando conciencia tanto de sus recursos básicos como de todo su cuerpo, prevalece en su mente la ecuación madre-comida; sin embargo, a medida que crece, se separa e independiza y adquiere una noción de individualidad corporal que lo convierte en un ser diferente y que modifica la ecuación antes establecida. Anna Freud (cf. Mahler) considera que mientras el niño se toma independiente frente al proceso de alimentacion, puede pasar por un conjunto de dificultades que pueden dejar una huella para una afección neurótica del estómago y del apetito en la vida adulta, especialmente si el niño creció en el marco de una relación (cuasi) simbiótica con su madre.

En la investigación de pacientes anoréxicas se ha observado que la madre se articula como una figura sobreprotectora que no puede concebir a su hijo como una persona libre e independiente con derechos propios y necesidades originales, lo cual determina que a lo largo de su desarrollo, el niño pierda la capacidad para reconocer la naturaleza y la legitimidad de su propias sensa- 
ciones y asocie sus necesidades y gratificaciones a las de sus padres. En este sentido, el niño pierde la posibilidad de compartir sus propias experiencias y necesidades, comienza a dudar de las mismas, se convierte en un ser sumiso y dependiente de las figuras parentales y pierde el sentido de su propio cuerpo. Para Mushatt (1982), la Anorexia Nervosa es un defecto en el desarrollo del Ego que se origina en una dificultad para resolver el problema de la separaciónindividuación; por lo tanto, es una falla para desarrollar el sentido de la individualidad y de la identidad afectiva y sexual.

La corriente sistémica (Selvini Palazzoli (1972); Minuchin, 1978) amplia el espectro de conceptualización de la enfermedad al proponer un enfoque interpersonal que complementa la perspectiva intrapsíquica de la corriente psicoanalítica. La Anorexia Nervosa se considera, desde esta perspectiva, como una enfermedad psicosomática que se gesta en el seno de una famiia particular en la cual el sujeto enfermo se articula como el "paciente identificado" portador del síntoma familiar. A su vez, la familia, a la que Minuchin (1978) considera como una "familia anoréxica" mantiene y respalda el síntoma en cuestión en la medida en que éste contribuye a sostener la homeostasis familiar. En este sentido, la unidad de análisis no es el paciente identificado sino el sistema familiar, en general, aunque se considera también como factible que el paciente identificado participe paralelamente de una terapia individual.

El sujeto anoréxico se caracteriza por presentar una baja deliberada de peso, la cual se ubica alrededor del $25 \%$ por debajo del peso considerado como normal de acuerdo a las tablas de talla y de peso. Se considera que existe una Anorexia Nervosa Primaria o Típica (Slade, 1984) cuando el sujeto enfermo tiene una actitud orientada sólo y únicamente hacia la comida, el control del peso y la imagen corporal y que existe una Anorexia Nervosa Secundaria o Atipica (Slade, 1984) cuando el dejar de comer no es un fin en si mismo sino, más bien, una consecuencia de cualquier otro problema psiquiátrico o físico. Por lo general, la Anorexia Nervosa se presenta en mujeres adolescentes con un alto coeficiente intelectual y un desempeño escolar notable. Son mujeres que se caracterizan por ser controladoras, minuciosas, meticulosas, capaces de vivir de acuerdo a las normas socio-culturales de su medio ambiente pero vacias a nivel afectivo y seguras de haber accedido a una forma de existencia física y moral superior en tanto que han logrado controlar su peso y su cuerpo.

Estos pacientes no son conscientes de su enfermedad. Mientras que ellos no reconozcan ni admitan su situación psíquico-física, cualquier intervención que provenga del medio respecto de su condición corporal y afectiva choca con una barrera defensiva impenetrable que se constituye a partir del (absurdo) convencimiento de que son saludables gracias a que controlan su peso y su cuerpo. La delusión corporal, que es una característica definitoria de la enfermedad como consecuencia de la inanición, refleja una disrupción del pensa- 
miento que, siguiendo a Slade (1984), puede estar vinculada a la disminución de la capacidad intelectual.

El temor a la gordura, la aparición de la crisis de la adolescencia tanto desde el punto de vista de los cambios físicos como desde la necesidad de asumir una identidad autónoma desligada de las figuras parentales, la incapacidad de comunicar adecuadamente la necesidad (cuasi obsesiva) de mantener ciertos controles y rutinas, la delusión de la imagen corporal, la baja autoestima, la necesidad de aislarse del medio social para vivir en un micromundo privado guiado por convicciones procedentes de la realidad distorsionada, la variación de los estados anímicos y la dificultad para separarse e individualizarse son las características más saltantes de la enfermedad. El tiempo de duración de la misma es variable, aunque en la mayoría de los casos la enfermedad dura un perfodo prolongado quizá porque el síndrome de la inanición instaura al sujeto en un circuito circular que llega a dominar su existencia.

La Anorexia Nervosa es un síndrome producto de la interacción y articulación en un tiempo definido de un conjunto de factores y fuerzas como son los valores y presiones de la sociedad occidental, la condición de jovenes adolescentes con presiones internas y externas para lograr el éxito frente al riesgo del anonimato y las características individuales y familiares específicas. Crisp (1970) considera que estos factores pueden remitirse incluso al período de embarazo de la madre. ${ }^{2}$ Minuchin (1978) por su parte, postula que la familia anoréxica es una familia psicosomática en la que se presentan alternativamente o en conjunto cuatro patrones típicos de funcionamiento: el aglutinamiento, la sobreprotección, la rigidez y la falta de capacidad para resolver conflictos. ${ }^{3}$

En las familias anoréxicas se ha observado que los padres son excesivamente sobreprotectores y que los pacientes identificados carecen de posibilidades suficientes para separarse e individualizarse; que la madre es, por lo general, una mujer excesivamente dominante y restrictiva y que el padre es

2. La alimentación de la madre durante el embarazo y las condiciones ambientales que la rodean pueden tener una repercusión directa en el peso del niño al nacer así como en sus tendencias alimenticias posteriores (Crisp, 1970).

3. El aglutinamiento se refiere a la extrema proximidad e intensidad con que se producen las interacciones familiares a nivel individual e intrasistémico. La sobreprotección se refiere a la preocupación excesiva que demuestran los diversos miembros de la familia entre si especialmente, los padres hacia los hijos. La rigidez se refiere a la necesidad de las familias de mantener su status y de no permitir cambios en el sistema. La falta de capacidad para resolver conflictos refleja la dificultad de estas familias para aceptar, enfrentar y resolver conflictos y la necesidad exagerada de vivir con la ilusión de que existe entre los miembros de la misma consenso y armonía. 
una persona exageradamente pasiva; que la relación entre los padres es hostil y que la hostilidad está siempre encubierta y que los padres diseñan un programa de disciplina muy rígido que exigen sea cumplido a cabalidad por sus hijos. ${ }^{4}$

Crisp (1970) señala que la enfermedad se gesta en la interacción hostil y dependiente del hijo (a) con sus padres antes que en una conflictiva individual específica del paciente identificado. En este sentido, se produce un giro en la compresión de la enfermedad: se pasa de una posición intrapsíquica a una posición interpersonal, giro que coincide con la complementariedad de los marcos sistémico y psicoanalítico.

Respecto a los aspectos demográficos de la enfermedad, en el Perú no contamos con una estadística de la casuística ya que no existe ni en los hospitales generales ni en las clínicas privadas servicios que registren y traten de una manera organizada y coherente la misma. Asi mismo, no contamos con investigaciones precedentes que revelen algún tipo de información específica. Sin embargo, estudios realizados en Estados Unidos e Inglaterra (Slade, 1984) revelan que una de cada 200 mujeres menores de 16 años y una de cada 100 mujeres mayores de 16 años adquieren la enfermedad; en las universidades, una de cada 50 mujeres; en las escuelas de danza y/o modelaje y en los departamentos de educación física, una de cada 14 mujeres y en las universidades y escuelas de arte dramático, una de cada 20 mujeres enferman de Anorexia Nervosa.

Se ha demostrado (Vandereycken \& Meermann, 1984) que la prevención primaria de la Anorexia Nervosa es todavía imposible ya que el foco de atención aún no puede ubicarse en las influencias causales que provienen de la sociedad; sin embargo, resultan factibles los niveles de prevención secundaria y terciaria ya que existen posibilidades de tratamientos individuales y familiares cuya finalidad es reduir, en la medida de lo posible, la cronicidad de la enfermedad y planificar estrategias de reducción de la misma en la comunidad a través de tratamientos útiles y variados.

De acuerdo a los criterios diagnosticos del DSM III los síntomas esenciales de la enfermedad son: el miedo intenso a la obesidad, la alteración de la imagen corporal, la pérdida significativa de peso de acuerdo a las tablas de talla y peso, la amenorrea, la dificultad para encontrar un correlato físico a los síntomas así como una explicación coherente a los mismos, la presencia de un

4. Se ha constatado que en las "familias anoréxicas" existe también una suerte de incomodidad social. Se trata de familias que han buscado ascender económicamente a estratos superiores casi a cualquier costo y que en el camino de reinserción no han podido manejar adecuadamente el conjunto de exigencias y presiones socio-culturales propias del nuevo medio, dejando de esta manera sembradas las semillas de probables problemas emocionales. 
conjunto de síntomas asociados, el retraso en el desarrollo psicosexual y un marcado desinterés por la actividad sexual. Slade (1984) añade que lo que diferencia a la Anorexia Nervosa de cualquier otro trastomo de la alimentación es la actitud que debe ser considerada como criterio diagnóstico.

\section{Metodología}

Atendiendo a la carencia de información respecto de la enfermedad en el Perú y a la cantidad de mujeres que enferman de la misma (una de cada 250 mujeres de acuerdo a los criterios del DSM III y una de cada 200 de acuerdo a las investigaciones de Slade) hemos considerado importante realizar una investigación como una primera aproximación a la realidad de la Anorexia Nervosa en nuestro medio. Esta se realizó a través del Método de Estudio de Casos Indivuales, método que nos permitió trabajar sumultáneamente los niveles intra e interpersonal en cada uno de los sujetos de la muestra. ${ }^{5}$ (Cuadro 1)

Cuadro 1: Características de la Muestra

\begin{tabular}{c|c|c|l|c|c|c|c}
\hline \multicolumn{2}{c|}{ Sexo } & Edad & Procedencia & Referencia & Peso & $\begin{array}{c}\text { Peso normal } \\
\text { de acuerdo a } \\
\text { tablas de } \\
\text { Talla/Peso }\end{array}$ & $\begin{array}{c}\% \text { peso } \\
\text { perdido }\end{array}$ \\
\hline 1 & F & 16 & Lima & INHD-HN & 38 & 56 & $31.2 \%$ \\
2 & F & 12 & Lima & $\begin{array}{c}\text { Pediatra } \\
\text { Privado }\end{array}$ & 28 & 42 & $33.4 \%$ \\
3 & F & 25 & Lima & $\begin{array}{c}\text { Medico General } \\
\text { Privado }\end{array}$ & 48.5 & 57 & $15 \%\left(^{*}\right)$ \\
4 & F & 26 & Lima & $\begin{array}{c}\text { Medico General } \\
\text { Privado }\end{array}$ & 45 & 57 & $21.1 \%\left(^{* *}\right)$ \\
\hline
\end{tabular}

Edad promedio: 19 años 9 meses (***)

Promedio peso perdido: $25.1 \%$

$\left(^{*}\right.$ El caso 3 se trata de una paciente anorexica/bulimica. La menor pérdida de peso se justifica por la ingesta compulsiva de alimentos.

(**) El caso 4 se trata de una pérdida inferior a $25 \%$ pero con una conducta que se identfica con las características de la anorexia nervosa primaria

(***) La anorexia nervosa se presenta con mayor influencia entre los 16 y 25 años.

5. En el Método de Estudio de Casos Individuales se trabaja a nivel intrapersonal e interpersonal: El primer nivel intenta un estudio exploratorio que pretende estudiar la personalidad del sujeto a través del conjunto de instrumentos estandarizados que pueden ser entrevistas, pruebas proyectivas y/o escalas psicológicas. El segundo nivel intenta una comparación de los resultados obtenidos a nivel intrapersonal con el fin de buscar la unidad en el interior de la diversidad y de encontrar las semejanzas y las diferencias para establecer la extensión en la cual los individuos comparten patrones de conducta que pueden ser identificados como tipos genuinos. 
Los instrumentos utilizados fueron elegidos en función de los factores estudiados. Los factores físicos fueron evaluados a partir de la entrevista individual y de la entrevista familiar, así como por las historias clínicas y los informes médicos. Los factores psicologicos individuales y/o familiares fueron evaluados tanto en las entrevistas individuales y/o familiares como por una batería de Pruebas Psicológicas compuestas por el Test de la Figura Humana, el Test de la Familia, el Test de Apercepción Temática (TAT), el Psicodiagnóstico de Rorschach y las Escalas de Inteligencia de Wechsler (WISC-R y WAIS). ${ }^{6}$ (Cuadro 2).

Cuadro 2: Factores Psicológicos Estudiados

\begin{tabular}{|c|c|c|c|c|c|c|c|}
\hline Factores & \begin{tabular}{|l|} 
Entrevista \\
Individual
\end{tabular} & $\begin{array}{l}\text { Entrevista } \\
\text { Farniliar }\end{array}$ & $\begin{array}{l}\text { Figura } \\
\text { Humana }\end{array}$ & $\begin{array}{c}\text { Test de la } \\
\text { Familia }\end{array}$ & TAT & $\begin{array}{l}\text { Psicodiagnóstico } \\
\text { de Rorschach }\end{array}$ & WiscW Wais \\
\hline \multicolumn{8}{|l|}{ Físicos } \\
\hline $\begin{array}{l}\text { Baja de peso } \\
\text { Amenorrea } \\
\text { Estreñimiento } \\
\text { Debilitamiento muscular } \\
\text { Hiperactividad } \\
\text { Edemas } \\
\text { Resistencia infecciones } \\
\text { Inanición/sex }\end{array}$ & $\begin{array}{l}x \\
x \\
x \\
x \\
x \\
x\end{array}$ & $\begin{array}{l}x \\
x \\
x\end{array}$ & & & & & \\
\hline \multicolumn{8}{|l|}{ Psicologicos } \\
\hline \multicolumn{8}{|l|}{ Individuales } \\
\hline $\begin{array}{l}\text { Temor a la gordura } \\
\text { Anorexia y adolescencia } \\
\text { Comunicación } \\
\text { Control } \\
\text { Disminución de la } \\
\text { Capacidad Intelectual } \\
\text { Baja autoestima } \\
\text { Imagen corporal } \\
\text { Aislamiento social } \\
\text { Estado de ánimo variable } \\
\text { Separación individuación }\end{array}$ & $\begin{array}{l}x \\
x \\
x \\
x \\
x\end{array}$ & $\begin{array}{l}x \\
x \\
x\end{array}$ & $\begin{array}{l}x \\
x \\
x \\
x \\
\\
x \\
x\end{array}$ & $\begin{array}{l}x \\
x \\
x \\
x \\
\\
x \\
x\end{array}$ & $\begin{array}{l}x \\
x \\
x \\
x \\
x \\
x \\
x \\
x\end{array}$ & $\begin{array}{l}x \\
x \\
x \\
x \\
x \\
x\end{array}$ & $\begin{array}{r}x \\
x \\
-x\end{array}$ \\
\hline \multicolumn{8}{|l|}{ Familiares } \\
\hline $\begin{array}{l}\text { Sobreprotección } \\
\text { Aglutinamiento } \\
\text { Rigidez } \\
\text { Incapacidad para resolver } \\
\text { confliclos }\end{array}$ & $\begin{array}{l}x \\
x \\
x \\
x\end{array}$ & $\begin{array}{l}x \\
x \\
x \\
x\end{array}$ & $x$ & $\begin{array}{l}x \\
\cdot x \\
x\end{array}$ & $\begin{array}{l}x \\
x \\
x \\
x\end{array}$ & $\begin{array}{l}x \\
x \\
x\end{array}$ & $x$ \\
\hline
\end{tabular}

6. La correlación entre el factor estudiado y las Pruebas Proyectivas se puede apreciar en el Cuadro

2. Es interesante anotar que un mismo factor fue evaluado por varias pruebas simultáneamente. 
En el nivel intrasujeto se trabaj6 con los criterios cuantitativo y cualitativo. El primero estuvo en relación a la calificación numérica de las pruebas psicológicas y el segundo estuvo en relación a la codificación e interpretación de estas pruebas. En el nivel intersujeto se hizo una comparación de la calidad de las respuestas de manera global, y del contenido de las mismas, en particular. Además, desde el punto de vista cuantitativo, se trabajaron los resultados utilizando la correlación biserial y de persona y el análisis factorial. (Para mayor información referirse a Zusman, L., 1989).

\section{Análisis de resultados y discusión}

Desde el punto de vista de los factores físicos, se observó que la salud de los sujetos anoréxicas antes de enfermar fue óptima. Al momento de la evaluación, el peso de las examinadas estaba entre el 15 y $25 \%$ por debajo del peso "normal" estipulado por las tablas de talla y de peso. La edad de aparición de la enfermedad en tres de las entrevistadas se presento entre los 14 y 18 años, rango de edad que estipula el criterio diagnóstico de DSM III. Sólo una de las examinadas presentó la enfermedad a los 11 años, siendo ésta una edad que también se contempla como factible (de acuerdo al mismo criterio diagnóstico), en la medida en que marca (aproximadamente) el fin de la etapa puberal y el ingreso a la adolescencia.

La voluntad férrea de no comer y/o de no engordar, la amenorrea, el estreñimiento, la resistencia a las infecciones y la hiperactividad son factores que se encontraron en las cuatro sujetos examinadas. La dificultad para enfrentar y enfrentarse a la sexualidad es un factor que se observo en las tres mayores y que no se manifestó en la joven de 11 años, quizá por que todavía no había ingresado a la adolescencia. A pesar de que en la literatura sobre Anorexia Nervosa se recalcan el debilitamiento muscular y los edemas como factores físicos típicos de la enfermedad, nosotros no lo hemos observado en los casos estudiados (Cuadro 3).

Las cuatro sujetos examinados fueron de sexo femenino; tres de ellas pertenecian a un sector socio-económico medio-alto y solo una a un sector socio-económico bajo, lo cual se vincula a la hipótesis de Slade (1984) que afirma que la población más numerosa de pacientes anoréxicos es la femenina proveniente de sectores economicos sociales altos, así como la hipótesis de Andersen \& Hay (1985) quienes afirman que también existen un número considerable de casos que provienen de los sectores menos favorecidos de la población. 
Cuadro 3: Resultados Individuales de cada caso

\begin{tabular}{|c|c|c|c|c|}
\hline Factores & 1 & 2 & 3 & 4 \\
\hline \multicolumn{5}{|l|}{ Físicos } \\
\hline $\begin{array}{l}\text { Baja de peso } \\
\text { Amenorrea } \\
\text { Estreñimiento } \\
\text { Debilitamiento Muscular } \\
\text { Hiperactividad } \\
\text { Edemas } \\
\text { Resistencia Infec. } \\
\text { Inanición/Sex }\end{array}$ & $\begin{array}{l}\mathbf{x} \\
\mathbf{x} \\
\mathbf{x} \\
\mathbf{x} \\
\mathbf{x} \\
\mathbf{x}\end{array}$ & $\begin{array}{l}\mathbf{x} \\
\mathbf{x} \\
\mathbf{x} \\
\mathbf{x} \\
\mathbf{x} \\
\mathbf{x}\end{array}$ & $\begin{array}{l}\mathbf{x} \\
\mathbf{x} \\
\mathbf{x} \\
\mathbf{x} \\
\mathbf{x} \\
\mathbf{x}\end{array}$ & $\begin{array}{l}\mathbf{x} \\
\mathbf{x} \\
\mathbf{x}\end{array}$ \\
\hline \multicolumn{5}{|l|}{ Psicológicos } \\
\hline \multicolumn{5}{|l|}{ Individuales } \\
\hline $\begin{array}{l}\text { Temor a la gordura } \\
\text { Anorexia y Adolescencia } \\
\text { Comunicación } \\
\text { Control } \\
\text { Disn. Cap. Intelectual } \\
\text { Baja autoestima } \\
\text { Imagen corporal } \\
\text { Aislamiento social } \\
\text { Estado de ánimo var. } \\
\text { Separación individual }\end{array}$ & $\begin{array}{l}x \\
x \\
x \\
x \\
x \\
x \\
x \\
x \\
x \\
x\end{array}$ & $\begin{array}{l}\mathbf{x} \\
\mathbf{x} \\
\mathbf{x} \\
\mathbf{x} \\
\mathbf{x} \\
\mathbf{x} \\
\mathbf{x} \\
\mathbf{x}\end{array}$ & $\begin{array}{l}\mathbf{x} \\
\mathbf{x} \\
\mathbf{x} \\
\mathbf{x} \\
\mathbf{x} \\
\mathbf{x} \\
\mathbf{x} \\
\mathbf{x} \\
\mathbf{x}\end{array}$ & $\begin{array}{l}\mathbf{x} \\
\mathbf{x} \\
\mathbf{x} \\
\mathbf{x} \\
\mathbf{x} \\
\mathbf{x} \\
\mathbf{x} \\
\mathbf{x}\end{array}$ \\
\hline \multicolumn{5}{|l|}{ Familiares } \\
\hline $\begin{array}{l}\text { Sobreprotección } \\
\text { Aglutinamiento } \\
\text { Rigidez } \\
\text { Incapacidad Res. conflictos }\end{array}$ & $\begin{array}{l}\mathbf{x} \\
\mathbf{x} \\
\mathbf{x} \\
\mathbf{x}\end{array}$ & $\begin{array}{l}\mathbf{x} \\
\mathbf{x} \\
\mathbf{x} \\
\mathbf{x}\end{array}$ & $\begin{array}{l}\mathbf{x} \\
\mathbf{x} \\
\mathbf{x} \\
\mathbf{x}\end{array}$ & $\begin{array}{l}\mathbf{x} \\
\mathbf{x} \\
\mathbf{x} \\
\mathbf{x}\end{array}$ \\
\hline
\end{tabular}

Desde el punto de vista de los factores psicológicos individuales se observó que las anoréxicas presentan una relación conflictiva con su cuerpo y con su imagen corporal. En ellas se pudo apreciar la negación de un cuerpo sumamente delgado, actitud que, en términos de Slade (1984) se denomina delusión corporal. En todas, se pudo apreciar que su cuerpo representa el espacio donde ellas depositan los contenidos agresivos y hostiles que no pueden canalizar a través de otras áreas o, en su defecto, sublimar. Asi mismo, tres de los cuatro casos examinados, manifestaron un deseo consciente de no 
engordar y de mantener un cuerpo esbelto y delgado. El caso restante negó la preocupación por la gordura y la esbeltez del cuerpo, actitud que, en términos de la evaluación global del sujeto, se entiende como una negación.

En dos de las examinadas, la enfermedad aparece durante el fin de la época y el ingreso a la adolescencia, pudiendo interpretarse que la Anorexia Nervosa se presenta como una formula que permite evadir la presencia de los cambios corporales, la inminencia de la sexualidad y las exigencias sociales, afectivas y culturales propias de la adolescencia. En las dos restantes, la enfermedad se presentó a los 18 años, cuando ellas abandonaron su hogar para iniciar una vida independiente. Garfinkel (1974) considera que cuando las jovenes salen de su hogar y se separan del sistema familiar se produce un quiebre tanto en la homeostasis de la familia como en el nivel intrapsíquico de los sujetos anoréxicas quienes, al no haber asimilado adecuadamente, los procesos de separación-individuación y no haber aprendido un modo de funcionamiento autónomo, se pierden dentro de un conjunto de exigencias, demandas y obligaciones que les impone su nueva realidad para las cuales no están preparádas. El quiebre de la homeostasis interna determina una regresión de características narcísisticas y la aparición de la Anorexia Nervosa.

Las evaluadas demostraron dificultad para comunicar y referirse a su enfermedad y a las características de la misma así como a los aspectos más conflictivos de su personalidad. Slade (1984) y Story (1982) coinciden en que "decodificar" un "mensaje anoréxico" a través del código comun de la sociedad es casi un imposible, por lo tanto, es necesario acceder a los rasgos más profundos de la cosmovisión de cada una de estas pacientes para comprender la realidad interna de las mismas.

Se observó también que la autoestima de las anoréxicas se vincula al estado de ánimo que ellas presentan en un momento determinado y que ambos factores dependen de la capacidad e incapacidad de ellas para controlar tanto la calidad de lo que ingieren como el peso que tienen.

El nivel intelectual de las sujetos anoréxicas se establece entre el nivel normal promedio y normal superior (CI: 108-116). A pesar de que sus aspiraciones intelectuales son elevadas, su funcionamiento intelectual se encuentra por debajo de su potencial, dada la interferencia de un conjunto de factores psicologicos de orden individual y familiar, asf como por la presencia de factores físicos que inciden en la disminución de la capacidad intelectual.

En relación a la capacidad de vincularse con el medio observamos la dificultad para establecer contactos adecuados y espontáneos con el medio así como una tendencia a negar o a escindir la realidad. Les resulta difícil esta- 
blecer compromisos estables con su medio y con los individuos que lo conforman y emiten respuestas superficiales, estereotipadas y aprendidas que las libera de la necesidad de establecer vínculos y compromisos afectivos. Presentan una tendencia a ser hostiles y agresivas con el medio y con quienes las rodean.

Las cuatro sujetos examinadas tuvieron a lo largo de su vida vínculos (cuasi) simbióticos con sus madres o, en su defecto, relaciones superyóicas; por lo tanto, se trata de mujeres que no han podido lograr la separación suficiente como para sentirse autónomas y efectivas. En este sentido, el cuerpo reducido a proporciones de un cuerpo pre-puberal representa el anclaje a la determinación inconsciente de no crecer.

Todos los casos provienen de hogares con problemas familiares severos. En estas familias se observo un exceso de aproximación entre los miembros por la falta de límite entre los mismos, la tendencia a la sobreprotección de los hijos, la rigidez como medio para mantener la homeostasis familiar, la incapacidad para evidenciar y resolver conflictos y la participacion de los hijos en el conflicto marital. Asi mismo, se observó la tendencia a la triangulación, es decir, la utilización del "paciente identificado" como un aliado de cada uno de los miembros de la pareja que se encuentra disociada o en conflicto.

De nuestras observaciones podemos concluir que la Anorexia Nervosa es una enfermedad determinada por un conjunto de factores psicologicos individuales y psicologicos familiares y/o por una combinación de los mismos. Cuando la psicopatología de la paciente está determinada por un conjunto de factores psicologicos individuales, su conducta tiende a ser más aislada y más retraída. Si su psicopatología está determinada por factores familiares, la conducta de la anoréxica es, más bien, abierta y compatible con el medio. La diferencia de estos dos modos de determinación de la conducta y de la personalidad nos permite plantear que existen casos de Anorexia Nervosa Estructural y casos de Anorexia Nervosa Reactiva.

Los procedimientos estadísticos aplicados indicaron que la Anorexia Nervosa se establece como una confluencia de factores psicologicos individuales y familiares. La enfermedad se presenta cuando los núcleos psicopatológicos hasta entonces reprimidos, emergen ante diferentes momentos criticos que el Yo no puede enfrentar como es el caso específico del ingreso a la adolescencia y a la aceptación de la realidad que ésta impone, como es, el ausmir la realidad de un cuerpo femenino y la sexualidad así como la necesidad de alejarse del medio familiar para asumir una posición autónoma e independiente. 
Nosotros consideramos que para el diagnóstico de la Anorexia Nervosa es necesario utilizar una batería completa de Pruebas Psicológicas que abarque tantos los aspectos psicologicos individuales como los familiares. Como pruebas aisladas, el diagnóstico resulta incompleto. Es importante resaltar la ausencia de contenidos orales en las Pruebas Gráficas y proyectivas y la dificultad para observar la mecánica de funcionamiento de la familia en la Prueba de Dibujo de la Familia. Esto nos demuestra la necesidad de observar más bien, el trasfondo vincular del síntoma antes que sus características aparentes.

\section{Referencias}

Andersen, A. \& Hay, A.: (1985). Racial and socioeconomic influences in anorexia nervosa and bulimia. International Journal of Eatine Disorders. Vol. 4, 4 pp. 479-487.

Brunch, H. (1974). Eating disorders, obesity and Anorexia Nervosa and the person within. Routledge and kegan Paul Ltd. Great Britain.

Brunch, H. (1981). Developmental considerations of Anorexia Nervosa and Obesity. Canadian journal of psychiatry. Vol. (26) pp. 212-217.

Brunch, H. (1982). Treatment in Anorexia Nervosa. International journal of psychoanalytic psychotherapy. Vol (9) pp. 303-312.

Crisp, A.H. (1970). Premorbid factors in adult disorders of weight, with particular reference to primary Anorexia Nervosa (weight phobia): a literature review. Journal of psychosonalic research. Vol. 14, pp. 1-22.

.Crisp, A.H. (1974a). Anorexia Nervosa: Psychoneurotic characteristic of parents: relationship to prognosis. Journal of psychosonatic research. Vol. (8) pp. 167-173.

Crisp, A.H. (1974b). Clinical and therapeutic aspects of Anorexia Nervosa: a study of 30 cases. Journal of psychosonatic research. Vol. (8) pp. 67.

Garfinkel, \& Gardner, D. (1982). Anorexia Nervosa: a multidementional perspective. Brunner, Matzel Publishers, $\mathrm{Ny}$

Hsu, G. (1983). The aetiology of Anorexia Nervosa. Psychological medicine. Vol. (13).

Mahler, M., Pine, F. \& Bergman, A. (1976). El Nacimiento Psicológico del Infante Humano. Simbiosis $e$ individuación. Buenos Aires: Marymar.

Minuchin, S. (1978). Psychosomatic families: Anorexia Nervosa in context. Harvard University Press.

Minuchin, S. (1986). Una familia anoréxica. Caleidoscopio familiar. Ediciones Paidos, pp. 88-107.

Mushatt, C. (1982). A psychoanalytic commentary of Anorexia Nervosa. International journal of psychoanalytic psychotherapy. Vol. (9), pp. 257265. 
Selvini Palazzoli, M. (1974). Self starvation: from the intrapsychic to the transpersonal approach to Anorexia Nervosa. London: Chauser publishing co. Ltd.

Slade, R. (1984). The anorexia nervosa reference book. London: Harper and Row Publishers.

Story, I. (1982). Anorexia Nervosa and the psychotherapeutic hospital. Journal of psychoanalytic psychotherapy. Vol (9), pp. 267-302.

Wilson, C. (1982). The fear of being fat and Anorexia Nervosa. International Journal of psychoanalytic psychotherapy. Vol. (9) pp. 234-255.

Vandereycken, W. \& Murmann, R. (1984). Anorexia Nervosa: is prevention possible. International journal of psychiatry in medicine. Vol. 14(3) pp. 191-201.

Zusman, L. (1988). Anorexia Nervosa:un estudio de cásos. Memoria de bachiller en Psicología, PUC. 